Science $\mathcal{W}$ Direct*

Cognitive Brain Research 23 (2005) 207-220
COGNITIVE

BRAIN

RESEARCH

www.elsevier.com/locate/cogbrainres

Research report

\title{
An event-related fMRI study of the neural networks underlying the encoding, maintenance, and retrieval phase in a delayed-match-to-sample task
}

\author{
Christian Habeck ${ }^{\mathrm{a}, *}$, Brian C. Rakitin ${ }^{\mathrm{a}, \mathrm{b}}$, James Moeller ${ }^{\mathrm{a}, \mathrm{c}}$, Nikolaos Scarmeas ${ }^{\mathrm{a}, \mathrm{b}, \mathrm{d}}$, Eric Zarahn ${ }^{\mathrm{a}, \mathrm{c}}$, \\ Truman Brown ${ }^{\mathrm{e}}$, Yaakov Stern ${ }^{\mathrm{a}, \mathrm{b}, \mathrm{c}, \mathrm{f}}$ \\ ${ }^{a}$ Cognitive Neuroscience Division of the Taub Institute for Research in Alzheimer's disease and the Aging Brain, 622 West 168th Street, \\ PH-18, New York, NY, USA \\ ${ }^{\mathrm{b}}$ Department of Neurology, College of Physicians and Surgeons of Columbia University, 630 West 168th Street, New York, NY, USA \\ ${ }^{\mathrm{c}}$ Department of Psychiatry, College of Physicians and Surgeons of Columbia University, 630 West 168th Street, New York, NY, USA \\ ${ }^{\mathrm{d}}$ Department of Medicine, College of Physicians and Surgeons of Columbia University, 630 West 168th Street, New York, NY, USA \\ ${ }^{\mathrm{e}}$ Department of Biomedical Engineering, College of Physicians and Surgeons of Columbia University, 630 West 168th Street, New York, NY, USA \\ ${ }^{\mathrm{f} D e p a r t m e n t ~ o f ~ P s y c h o l o g y, ~ C o l l e g e ~ o f ~ P h y s i c i a n s ~ a n d ~ S u r g e o n s ~ o f ~ C o l u m b i a ~ U n i v e r s i t y, ~} 630$ West 168th Street, New York, NY, USA
}

Accepted 20 October 2004

Available online 9 December 2004

\begin{abstract}
Memory loads exceeding the limited capacity of working memory (WM) have been shown to expand the prefrontal areas that participate in WM and have revealed substantial individual differences in performance. We used a delayed-match-to-sample (DMS) task in an eventrelated fMRI study to map the full extent of the expanded regional activations associated with supracapacity loads. A 6-letter study array was compared to arrays of 1 and 3 letters. The task comprised separate encoding, retention, and retrieval fMRI epochs. A brain-wide spatial covariance analysis was applied to the data of all task epochs to identify patterns of correlated regional activations whose expression increased monotonically across 3 memory-load levels on a subject-by-subject basis. Such load-related activation patterns were in all task phases. Of greatest interest is the activation pattern that was obtained during the maintenance phase: increasing activation with memory load was found not only in the lateral PFC (BA 9,44) but also in the parietal lobe (BA 7,40), anterior cingulate (BA 32), and cerebellum. Decreasing activation was found in the occipito-temporal lobe (BA 19,39) as well as the medial prefrontal cortex (BA 9,10). Subject increases in pattern expression from 1 to 6 items were positively correlated with the corresponding reaction time increases $(p<0.05)$ and negatively correlated with NARTIQ $(p<0.05)$, indicating that people who were faster in their responses and had higher NARTIQ had to increase their subject expression of the memory-load-related activation pattern less and were more efficient at the cognitive task. Our method thus not only reproduced findings of other WM studies but also addressed the issue of interactions between lateral PFC and other parts of the brain during the task, for the retention of the to-be-remembered information. The load-related activation patterns from encoding and retrieval phase and their relationship to behavior are also discussed.
\end{abstract}

(C) 2004 Elsevier B.V. All rights reserved.

Theme: Neural basis of behavior

Topic: Cognition

Keywords: Covariance analysis; Functional imaging; Neural networks; Verbal recognition memory

\section{Introduction}

* Corresponding author. 630 West 168th Street, P and S Box 16, New York, NY, 10032, USA. Fax: +1 2123421838.

E-mail address: ch629@columbia.edu (C. Habeck).

The brain consists of a set of functionally distinct regions, yet these regions are also interconnected to form functional networks. In the study reported here, we sought 
to identify functional networks that support basic encoding, maintenance and retrieval mechanisms in a working memory (WM) task, and that display a load-related modulation with the amount of the to-be-remembered information. With this goal in mind, we have chosen to examine the MR signal during performance of a speeded short-term memory task descended from the Sternberg paradigm. In addition to basic visual, motor, and short-term memory function, this paradigm also provides an internal manipulation of difficulty that is useful in addressing the specificity of the observed brain networks.

We adopted a delayed-match-to-sample (DMS) task that was a variant of the Sternberg memory-scanning task. This short-term memory task offers a manipulation of difficulty in terms of memory load and allows precise chronometric information on subjects' performance. Our version of the task contains an encoding phase ("stimulus"), maintenance phase ("retention"), and a retrieval phase ("probe"). This short-term memory task has been studied extensively inside and outside the scanner (e.g., Refs. [23,27,31,32]), is easy to administer, and taps a set of cognitive component processes (pertaining to visual encoding, rehearsal, memory scanning, binary decision, response selection, and motor output). Advances in event-related imaging techniques have facilitated the separation of the different cognitive processes involved in task performance into different temporal components $[7,23,28]$. Of particular interest has been the role of the lateral prefrontal cortex (LPFC) and its relation to the number of items that have to be retained in the task ("memory load"). A recent eventrelated study [28] of a DMS task with serial presentation of 1 to 8 letters found decreasing activation in the ventral portion of the LPFC (BA 44,45,47) with increasing memory load during encoding, whereas the dorsal portion of the LPFC (BA 9,46) displayed increasing activation with increasing memory load during encoding. Consistent with this finding are the reports of different roles of ventral and dorsal portions of the LPFC: dorsal LPFC becomes involved once the quantity of the to-be-remembered information exceeds the working-memory capacity limit of " $4 \pm 1$ items" [5], necessitating executive "chunking" processes to encode information successfully. Presumably, the ventral portion of the LPFC, which is involved with maintenance and rehearsal processes at below-capacity levels, will then decrease its involvement.

The LPFC presents a nuanced picture with differences in the directionality of activation and its implication for task performance, depending on the task component analyzed, and the relationship with memory load. On the one hand, greater load-dependent activation during the retention period was beneficial for task-performance for both LPFC areas; that is, the more subjects activated dorsal LPFC and ventral LPFC during the retention period in a load-dependent manner, the more accurately and faster they classified the probe letter. On the other hand, overall activation (regardless of memory load) in the LPFC during the encoding and retrieval periods was lower for subjects that performed faster and more accurately.

We set out to find a pattern of brain regions whose activation shows a consistent relationship to memory load with a spatial covariance technique for a sufficiently large number of subjects. Questions that have been less emphasized in the past but deserve more exhaustive analysis in our view are the following: (1) what are the areas other than lateral prefrontal cortex that show a consistent relationship with memory load? (2) Is there deactivation in any areas in response to increasing memory load?

Multivariate analyses, which utilize the correlation of activation in different brain areas, can address both of these points. They identify a set of regions whose covarying activation and deactivation is associated with memory load. Regions showing an increasing activation with increasing memory load can be interpreted to reflect the greater demands of processing. Regions showing decreasing activation with increasing memory load might signal a suppression of activity brought by monosynaptic inhibitory influences of the regions showing increased activation. A further possibility is the phenomenon of "resource depletion" [9], i.e., a shift of resources away from ongoing, but inessential, processes to an increasingly demanding cognitive task: although the areas showing decreased activation might not be functionally connected to the areas increasing in activation in response to increasing memory load, the brain might have to devote more resources to the encoding, maintenance, and retrieval of the to-be-remembered information, and consequently decreases the level of activity in areas not crucial for the task at hand. The amount of this shift might still be subject-dependent, with a fixed ratio between activity increases and decreases, and result in a large covariance between areas detectable by a multivariate analysis technique.

Our current study therefore aims to integrate some of the findings regarding the involvement of the lateral prefrontal cortex in verbal working memory, while extending our analysis to every region in the brain.

\section{Methods}

Forty healthy subjects (age $=25.02 \pm 3.68$ years) participated in an event-related functional magnetic resonance imaging (efMRI) paradigm of a delayed-match-to-sample (DMS) task. All subjects were right-handed and carefully screened to ensure that they had no history of medical, psychiatric, neurological, or sleep disorders. Informed consent, as approved by the Internal Review Board of the College of Physicians and Surgeons of Columbia University, was obtained prior to study participation and after the nature, and risks of the study were explained. Subjects were paid for their participation in the study. 


\subsection{Delayed-match-to-sample task}

The DMS task was a variant of the Sternberg task [31,32]. A trial lasted a total $16 \mathrm{~s}$. Subjects were instructed to respond as accurately as possible. No feedback about their performance was given. The sequence of trial events was as follows (Fig. 1): first, a fixed 3-s period of blank presentation marked the beginning of trial; then, during the stimulus period of the task, an array of 1,3 , or 6 capital letters were presented for $3 \mathrm{~s}$ ("stimulus phase").

With the offset of the visual stimulus, subjects were instructed to focus on the blank screen and hold the stimulus items in mind for a 7-s maintenance interval ("retention phase"). Finally, a probe appeared for 3-s ("probe phase"), which was a lowercase letter centered in the field of view. In response to the probe, subjects indicated by a button press whether or not the probe matched a letter in study array (left index finger indicated "Yes", right index finger indicated "No").

Each experimental block contained 10 trials for each of three set sizes with five true negative and five true positive probes per set size. Three experimental blocks were run in total, yielding $10 \times 3 \times 3=90$ experimental trials per scanning session. In addition to the fixed 3 -s period of a blank screen presentation, which we counted as part of the experimental trial, there were intertrial intervals (ITI) that consisted of presentation of a blank screen and were used as baseline epochs in the time series analysis of the subject's data. Their length was variable and determined in the following way: 70 2-s increments were available throughout the whole block for 30 intertrial intervals. It was decided stochastically whether a 2-s increment of ITI would be inserted prior to the start of the trial, or whether the trial would begin immediately. The probabilities for choosing between additional ITI increments or starting the experimental trial were updated according to a sampling-without-replacement rule: for example, at the start of the experimental block, the

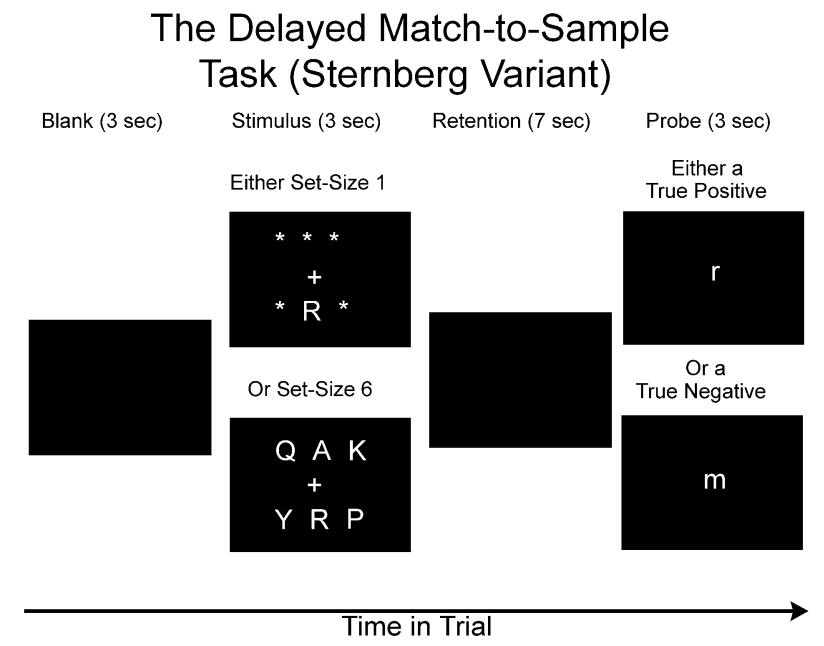

Fig. 1. Schematic sketch of delayed-match-to-sample paradigm of the Sternberg variant. probabilities for choosing a 2-s ITI increment vs. starting the experimental trial were $70 / 100$ vs. $30 / 100$. If a 2 -s ITI increment were chosen, these probabilities would be updated to $69 / 99$ vs. $30 / 99$ for the decision of adding another ITI increment vs. starting the trials. A further decision might reject the addition of yet another 2-s increment, which terminates the sampling process for this ITI. All together, the total ITI in this case would be $2 \mathrm{~s}$, and 69 2-s increments are still left for distribution throughout the remainder of the block. This sampling rule keeps the overall time assigned to ITI constant at $70 \times 2=140 \mathrm{~s}$ per block. With 30 trials of $16 \mathrm{~s}$ each, each block therefore lasted for $140+(30 \times 16)=620 \mathrm{~s}$. There were two breaks of a minute each between block 1 and 2 as well as block 2 and 3. This brings the overall time subjects spent in the scanner by each subject to $(3 \times 620)+120=1980 \mathrm{~s}$ or $33 \mathrm{~min}$.

Subjects went through a training run of seven blocks on the evening prior to the start of the experiment, the first six of which were administered with feedback. The training session was conducted to eliminate confounding effects from learning through repeating the performance of the DMS task.

\subsection{Behavioral and neuropsychological measures}

Performance variables were the (within-subject) mean reaction time as well as (within-subject) reaction time variability. Reaction time variability was computed as the coefficient of variation (CV), i.e., the standard deviation divided by the mean in subjects' reaction times for a particular number of study items. The rationale for this measure was to capture variability that was not explained by a scaling relationship with mean reaction time alone. Recognition accuracies and nonresponse rates were recorded too but were not used for any brain-behavior correlational analysis since they did not differ as a function of memory-load level (see below). Furthermore, subjects performed the New Adult Reading Test (NART)-North American version-prior to the DMS task. Its score was used for correlational analysis and denoted as NARTIQ.

Rather than the performance variables reaction time and reaction time variability per se, we used their load-related increases from 1-item to 6-item trials for the brain-behavior analysis. These two quantities combined with NARTIQ did not show any significant mutual significant correlation (maximum value of these three correlations: $R^{2}<0.09$, $p=0.06)$.

\subsection{FMRI acquisition and processing}

Functional images were acquired using a 1.5 Tesla magnetic resonance scanner (Philips). A gradient echo EPI sequence $\left(\mathrm{TE}=50 \mathrm{~ms} ; \mathrm{TR}=3 \mathrm{~s}\right.$; flip angle $\left.=90^{\circ}\right)$ and a standard quadrature head coil was used to acquire $\mathrm{T} 2 *$ weighted images with an in-plane resolution of $3.124 \times 3.124 \mathrm{~mm}$ (64×64 matrix; $20 \mathrm{~cm}^{2}$ field of view). Based on $\mathrm{T}_{1}$ " scout" 
images, $8 \mathrm{~mm}$ transaxial slices (15-17) were acquired. Following the fMRI runs, a high (in-plane)-resolution $\mathrm{T}_{2}$ image at the same slice locations used in the fMRI run was acquired using a fast spin echo sequence $(\mathrm{TE}=100 \mathrm{~ms}$; $\mathrm{TR}=3 \mathrm{~s} ; 256 \times 256$ matrix; $20 \mathrm{~cm}^{2}$ field of view). Task administration and data collection were controlled by a computer running appropriate software (Psyscope 1.1) and electronically synchronized with the MR scanner. Task stimuli were back-projected onto a screen located at the foot of the MRI bed using an LCD projector. Subjects viewed the screen via a mirror system located in the head coil. Task responses were made on a LUMItouch response system, and behavioral response data were recorded on the task computer.

All image processing and analysis was done using the SPM99 program (Wellcome Department of Cognitive Neurology) and other code written in Matlab 5.3 (Mathworks, Natick MA). FMRI time series were corrected for order of slice acquisition. All functional volumes in a given subject were realigned to the first volume from the first run of each study. The $\mathrm{T}_{2}$ anatomical image was then coregistered to the first functional volume, using the mutual information coregistration algorithm implemented in SPM99. This coregistered structural image was then used in determining nonlinear spatial normalization $(7 \times 8 \times 7$ nonlinear basis functions) parameters for a transformation into a Talairach standard space defined by the Montreal Neurological Institute template brain applied with SPM99. These normalization parameters were then applied to the functional data (using SINC-interpolation to reslice the images to $2 \times 2 \times 2 \mathrm{~mm}$ ).

\subsection{First-level analysis}

The fMRI responses to the three separate temporal components of the task, in each experimental condition and in each block, were fit to separate sets of predictor variables [34]. The predictor variables that were ultimately used in the first-level model estimation were obtained in the following way: a constant intercept was chosen for the all task phases. For one block, this results in three predictor variables ( 1 for stimulus, 1 for retention, 1 for probe) per set size (1,3, and 6) per probe type (positive or negative). An additional intercept term is provided for the effect of block, bringing the total number of predictor variables per block to $(3 \times 3 \times 2)+1=19$. Predictor variables had a nonzero value at every point in the time series where a particular condition was met and a zero value at every other point. For example, one predictor had a value of 1 during all stimulus phases of set size 1, with a positive probe, during the first block. Nonresponse trials, i.e., trials for which the subject missed the 3-s deadline for a response in the probe period, were excluded from any model estimation.

The set was convolved with a canonical hemodynamic response waveform (a sum of two gamma functions, as specified in the SPM99 program [10]) whose beginnings were marked by the appropriate onset vector for each epoch, set size, and probe type. The resulting time series vectors were used in the design matrix for the withinsubject model estimation. The final matrix had 3-by-3 block-diagonal form. The number of rows was the total number of volumes denoting the complete fMRI time series across the scanning session. The number of columns was $3 \times 19=57 ; 19$ design vectors for each experimental block, as explained above.

The band-pass filtered (low pass by a Gaussian with a FWHM of $4 \mathrm{~s}$ and a high pass cutoff of $14.5 \mathrm{mHz}$ ) fMRI time series at each voxel were regressed onto these predictor variables. A first-order autoregressive autocorrelation model was fit to the residuals to make statistical inference more robust to the intrinsic temporal autocorrelation structure [11].

At every voxel in the image, contrasts assessed the amplitudes (regression coefficients) of the components of the event-related responses that matched the canonical hemodynamic response waveform for the whole scanning session. A typical contrast used in our analysis for instance would be "activity during the retention phase for 6 items collapsed across probe types and experimental blocks vs. activity in the initial 3-s blank-period of that trial". This method of time-series modeling and contrast estimation at each voxel reduces the number of images to 1 per subject per condition. To account for gain differences between fMRI sessions, activation values were normalized by their voxel averages. The resulting parametric map images were smoothed using an isotropic Gaussian kernel (FWHM=8 $\mathrm{mm}$ ) and used as the data in the subsequent analysis. In total, there were nine parametric maps of normalized regression weights for each subject: 1 for each of 3 memory-load levels (1/3/6 letters) for each of three task phases (stimulus/retention/probe). These parametric maps serve as the dependent variables for the subsequent population-level multivariate analysis.

\subsection{Second-level multivariate analysis}

Ordinal Trend Canonical Variates Analysis (OrT CVA) $[12,13]$ was performed on the data. This analysis is similar to other regional covariance analyses techniques, notably Partial Least Squares, to the extent that it applies principal components analysis (PCA) to the data matrix that is transformed using a matrix representing the experimental design [20,33]. OrT CVA was designed to identify a covariance pattern in the MR signal whose expression increases across memory load levels $1 / 3 / 6$ for as many subjects as possible. Such a covariance pattern can be produced from a linear combination involving a small set of principal components. The coefficients for this linear combination are obtained through a multiple linear regression, aiming for an activation of the resulting pattern that best approximates a linear mean trend across the memoryload levels. 
A multivariate approach does not focus on any particular brain region (i.e., like DLPFC) and its associated task-related activation but proceeds in a brain-wide manner without any prior assumptions about brain localization. On the other hand, our analysis tests for types of activation with stringent constraints: patterns of sustained functional connectivity whose individual subject expression follows the experimental parameter (memory load) monotonically. By incorporating this constraint of a monotonic relationship with memory load on a subject-by-subject basis, we can increase the confidence of finding highly specific neural correlates of load-dependent encoding, retention, and retrieval processes. Our analysis should thus give a more complete picture of how these processes are implemented in the brain.

Multivariate analysis of multiple task conditions can proceed in a variety of ways: one could arrive at several covariance patterns -1 per condition - by analyzing the across-subject variances separately for each condition. This allows one to establish differences between task conditions concerning: (1) the brain areas involved, i.e., brain area $X$ 's activation might account for a lot of the variance in task condition 1 but not play a significant role in task condition 2 , and vice versa; (2) a changing strength of the correlation between brain areas that are involved in both tasks, i.e., brain areas $X$ and $Y$ show a correlation in their activation across subjects of $R=0.8$ during task condition 1 , but this correlation reduces and switches directionality to $R=-0.2$ during task condition 2.

On the other hand, data from multiple task scans can be analyzed such that one covariance pattern reveals interesting similarities or differences between conditions. For an example of the latter approach, readers are advised to consult Ref. [21]. We chose the former approach: rather than examining differences in functional connectivity between conditions, we were interested in changes in regional activation induced by increasing memory load that keep the functional connectivity unchanged across load levels and can therefore be captured in one covariance pattern. In our case, a single covariance pattern represents a set of brain regions whose mutual connections are not changing. Rather, the change across task conditions in the degree of expression of this covariance pattern varies from subject to subject. In addition, our technique is designed to find a pattern with an increase in expression across load levels for as many subjects as possible. This means that most people are showing the same mutually correlated regional activation and deactivation in response to memory load increases, with individual differences in the degree of these changes.

The goal of our analysis method can therefore be interpreted as finding 'quantitative' changes or 'changes in degree' in response to increasing memory load. A simple analogy that employs Euclidean geometry can aid the reader's visualization here: a single covariance pattern denotes one preferred direction in the high-dimensional vector space that is spanned by every voxel's activation value. Subjects' neural responses to increasing memory load can be seen as moving along this preferred direction, with individual differences in the amount of movement. 'Qualitative' changes or 'changes in kind' might be construed as changes in the preferred direction itself in response to increasing memory load, which happens if the correlative relationships between voxels' activation values themselves change. We decided to focus on the former objective first since we believe that identifying invariant aspects of functional connectivity has a better prospect for replication beyond the particular study under consideration than focusing on alterations in functional connectivity. Alterations in functional connectivity are addressed by other multivariate analysis methods such as Partial Least Squares $[19,20]$ and can be seen as complementary to the objective of our analysis method.

Individual subject's expression of the activation pattern, i.e., in terms of the previous analogy, this would be the amount of movement along the preferred direction, is quantified with the subject-scaling factor (SSF). The SSF is obtained by the operation of an inner product (=covariance across brain regions) between the covariance pattern in question and a subject's task scan. It quantifies to what extent a subject expresses the activation pattern in a task scan with a single number, which can be used for further analysis.

The property of a consistent monotonic within-subject change of pattern expression across task conditions (beyond mere mean change) is called an "ordinal trend" and quantified with the repeated-measures $F$-statistic ("rm$\left.F^{\prime \prime}\right)$. The set of principal components that was used to make up the activation pattern needs to be taken into consideration here too since using more principal components to produce a linear combination pattern to fit a particular expression behavior will result in bigger $p$ values. For equal values of the rm- $F$ statistic, a covariance pattern that was produced from principal components 1 and 2, for instance, is therefore less likely to have been obtained by chance than a pattern that was made up from principal components $1-5$.

A random permutation test generates the $p$-level for the rm- $F$ statistic actually observed for the activation pattern in our experimental sample. For these permutations, we analyzed the resampled data at least 1000 times with random subject-condition assignments, employing exactly the same set of principal components that was recruited in the linear combination to produce the activation pattern in our subject sample. The fraction of these analyses of the resampled data that exceeded the rm- $F$ statistic obtained in the point estimate could be interpreted as a $p$-level. A significant ordinal trend lends additional credence to the claim that an activation pattern was obtained through the experimental design manipulation rather than a statistically significant mean change mean across conditions that might have come about purely as a result of overly influential 
subject outliers. This added that across-subject consistency improves the sensitivity of the method without any sacrifice of specificity.
Activation patterns resulting from multivariate analysis assign different weights to all voxels included in the analysis, depending on the salience of their covariance

Table 1

Talairach locations of brain regions with significant activation or deactivation across load levels during the stimulus phase, as ascertained by a bootstrapresampling test $(|z|>2.33)$

\begin{tabular}{lrrlll}
\hline$X$ & $Y$ & $Z$ & Lobe & Structure & Brodmann label \\
\hline Positive weights & & & & & $*$ \\
4 & -80 & 49 & Occipital lobe & Lingual gyrus & Brodmann area 6 \\
-2 & 10 & 34 & Frontal lobe & Superior frontal gyrus & Brodmann area 32 \\
-2 & 15 & 9 & Frontal lobe & Cingulate gyrus & $*$ \\
4 & -5 & 46 & Sublobar & Thalamus & Brodmann area 7 \\
-2 & -72 & 31 & Parietal lobe & Precuneus & Medial dorsal nucleus \\
2 & -30 & 12 & Limbic lobe & Cingulate gyrus & $*$
\end{tabular}

Negative weights

\begin{tabular}{|c|c|c|c|c|c|}
\hline 36 & -22 & 18 & Sublobar & Insula & Brodmann area 13 \\
\hline-30 & -43 & -3 & Limbic lobe & Parahippocampal gyrus & Brodmann area 19 \\
\hline-10 & -47 & -11 & Anterior lobe & Cerebellar lingual & $*$ \\
\hline 67 & -33 & -8 & Temporal lobe & Middle temporal gyrus & Brodmann area 21 \\
\hline 59 & -26 & -10 & Temporal lobe & Middle temporal gyrus & Brodmann area 21 \\
\hline 65 & -47 & 1 & Temporal lobe & Middle temporal gyrus & Brodmann area 21 \\
\hline-22 & 33 & 39 & Frontal lobe & Middle frontal gyrus & Brodmann area 8 \\
\hline-24 & 44 & 25 & Frontal lobe & Superior Frontal gyrus & Brodmann area 10 \\
\hline-40 & -13 & 10 & Sublobar & Insula & Brodmann area 13 \\
\hline-36 & -30 & 16 & Temporal lobe & Superior temporal gyrus & Brodmann area 41 \\
\hline 42 & -36 & -22 & Temporal lobe & Fusiform gyrus & Brodmann area 36 \\
\hline-51 & -40 & -22 & Temporal lobe & Fusiform gyrus & Brodmann area 20 \\
\hline-38 & -30 & -20 & Limbic lobe & Parahippocampal gyrus & Brodmann area 36 \\
\hline-65 & -30 & -10 & Temporal lobe & Middle temporal gyrus & Brodmann area 21 \\
\hline-65 & -39 & -8 & Temporal lobe & Middle temporal gyrus & Brodmann area 21 \\
\hline-10 & -49 & 30 & Parietal lobe & Precuneus & Brodmann area 31 \\
\hline 24 & 27 & 41 & Frontal lobe & Middle frontal gyrus & Brodmann area 8 \\
\hline 32 & -43 & -6 & Limbic lobe & Parahippocampal gyrus & Brodmann area 19 \\
\hline-26 & 30 & -13 & Frontal lobe & Inferior frontal gyrus & Brodmann area 47 \\
\hline-44 & -74 & 30 & Temporal lobe & Angular gyrus & Brodmann area 39 \\
\hline 14 & -25 & 40 & Limbic lobe & Cingulate gyrus & Brodmann area 31 \\
\hline 14 & -7 & -20 & Limbic lobe & Uncus & $*$ \\
\hline 61 & -29 & 38 & Parietal lobe & Postcentral gyrus & * \\
\hline-59 & -58 & 10 & Temporal lobe & Middle temporal gyrus & $*$ \\
\hline 14 & 9 & 16 & Sublobar & Caudate & Caudate body \\
\hline 14 & 18 & 8 & Sublobar & Caudate & Caudate body \\
\hline 65 & -34 & 22 & Parietal lobe & Inferior parietal lobule & * \\
\hline 16 & -1 & -22 & Limbic lobe & Uncus & Brodmann area 34 \\
\hline-40 & -42 & -21 & Cerebellum & Culmen & $*$ \\
\hline 14 & 9 & -16 & Frontal lobe & Medial frontal gyrus & $*$ \\
\hline 6 & -50 & -19 & Cerebellum & Fastigium & $*$ \\
\hline 4 & -10 & 30 & Limbic lobe & Cingulate gyrus & Brodmann area 24 \\
\hline-44 & -48 & -25 & Cerebellum & Culmen & * \\
\hline-63 & -50 & 10 & Temporal lobe & Superior temporal gyrus & Brodmann area 22 \\
\hline 16 & -21 & 7 & Sublobar & Thalamus & Ventral posterior medial nucleus \\
\hline-6 & 9 & -17 & Frontal lobe & Medial frontal gyrus & Brodmann area 25 \\
\hline 63 & -51 & -6 & Temporal lobe & Middle temporal gyrus & Brodmann area 21 \\
\hline 65 & -14 & -3 & Temporal lobe & Middle temporal gyrus & Brodmann area 21 \\
\hline 4 & 17 & 23 & Limbic lobe & Anterior Cingulate & Brodmann area 24 \\
\hline-12 & 12 & 12 & Sublobar & Caudate & Caudate body \\
\hline-6 & 15 & -4 & Sublobar & Caudate & Caudate head \\
\hline-4 & -12 & 1 & Sublobar & Thalamus & $*$ \\
\hline-42 & -81 & 19 & Temporal lobe & Middle temporal gyrus & Brodmann area 19 \\
\hline 24 & -5 & -22 & Limbic lobe & Uncus & Amygdala \\
\hline
\end{tabular}

White matter locations have been removed. Top: positive weights, indicating activation across load levels for the majority of subjects; bottom: negative weights, indicating deactivation. 
contribution. Positive voxel weights indicate a positive correlation between the subject expression value and the associated regional activation, whereas negative weights indicate a negative correlation. This means that as the expression of a pattern increases, activation in the positively weighted regions increases as well, whereas activation in the negatively weighted regions decreases. The absolute magnitude of a regional weight determines the slope of this change: for instance, a region whose weight is twice as large as that of another also changes its activation twice as steeply. Whether a voxel weight is reliably different from zero is assessed by a bootstrap estimation procedure [8]. Denoting the point estimate of a voxel weight as $w$ and the standard deviation resulting from the bootstrap resampling procedure as $s_{\mathrm{w}}$, we can

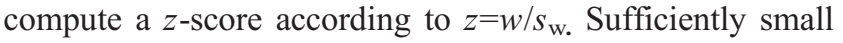
variability of a voxel weight around its point estimate value in the resampling processes results in a $z$-value of large magnitude and indicates a reliable contribution to the covariance pattern. As the threshold criterion, we chose $|z|>2.33$; under the assumptions of a standard-normal distribution, i.e., $z \sim N(0,1)$, this corresponds to a one-tailed probability of 0.01 .

Once an activation pattern was identified that systematically increased in expression as a function of memory load, we examined the correlation between individual change in network expression from 1 to $6[\operatorname{SSF}(6)-\operatorname{SSF}(1)]$ and the corresponding change in their scores on the task performance measures as well as neuropsychological measures collected outside the scanner.

\section{Results}

\subsection{Behavioral performance}

We included (within-subject) mean reaction times as well as (within-subject) coefficient of variation $(\mathrm{CV})$ in reaction time in our behavioral outcome measures. Reaction times showed a load-dependent increase: $\mathrm{RT}(1)=847 \pm 170 \mathrm{~ms}$, RT(3) $=1015 \pm 202, \mathrm{RT}(6)=1148 \pm 227 \mathrm{~ms}$ (H-F corrected $\left.\mathrm{rm}-F_{2,78}=106.18, p<0.0001\right)$, variability in reaction time was biggest for the 3-item array: CV RT(1) $=0.305 \pm 0.117$, CV RT(3) $=0.348 \pm 0.085$, CV RT(6) $=0.299 \pm 0.092$ (H-F corrected $\left.\mathrm{rm}-F_{2,78}=4.01, p<0.05\right)$. Recognition accuracies stayed constant across memory load: $\mathrm{P}(1)=98 \pm 3 \%, \mathrm{P}(3)=$ $97 \pm 5 \%, \mathrm{P}(6)=97 \pm 4 \% \quad\left(\mathrm{H}-\mathrm{F}\right.$ corrected $\mathrm{rm}-F_{2,78}=1.61$, $p=0.20)$. The fraction of nonresponse trials stayed constant too, $\mathrm{NR}(1)=1 \pm 4 \%, \mathrm{NR}(3)=1 \pm 2 \%, \mathrm{NR}(3)=1 \pm 2 \%$ (H-F corrected $\left.\mathrm{rm}-F_{2,78}=0.78, p=0.39\right)$.

\section{2. $f M R I$ data}

For the purposes of the MR image analysis, our experiment contained the main design parameter of memory load $(1,3$, or 6 items). For each task phase, we subjected the data for set size conditions 1,3 , and 6 to an Ordinal Trend Analysis.

In the following, we discuss the results of the analyses of separate task phases in the order of their administration: first stimulus, then retention, then probe. Using the first two principal components of the stimulus phase data, we found an activation pattern that showed a monotonic increase in expression across set sizes (permutation test: rm$F=42.7730, p<0.001$ ). This pattern accounted for $20 \%$ of the variance in the data. Areas of that showed robust $z$ scores in the bootstrap resampling process were found in occipital, temporal, parietal, limbic, and thalamic areas (see detailed listing for both increased and decreased activation in Table 1).

Areas that exclusively deactivated across set size were found in the cerebellum and basal ganglia (caudate). No significant correlation between this activation pattern's subject expression and behavioral performance variables could be found.

We then focused in more detail on the retention phase. Because it did not contain any stimuli or behavioral responses, it presents the best opportunity to approach pure rehearsal processes operating during working memory and identify their neural correlates. When applied to the data from the retention phase, our analysis produced significant results: a linear combination of the first two principal components produced by OrT CVA-displayed ordinal trend properties (permutation test: $\mathrm{rm}-F=38.6575, p<0.001$ ), suggesting an unambiguous memory load-related neural correlate of rehearsal. This pattern accounted for $9 \%$ of the variance in the data (Fig. 2).

Brain regions that concomitantly increased in activation (as ascertained by the bootstrap test) for the majority of subjects were found mainly in lateral PFC (BA 9,44), the parietal lobe (BA 7,40), anterior cingulate (BA 32), and

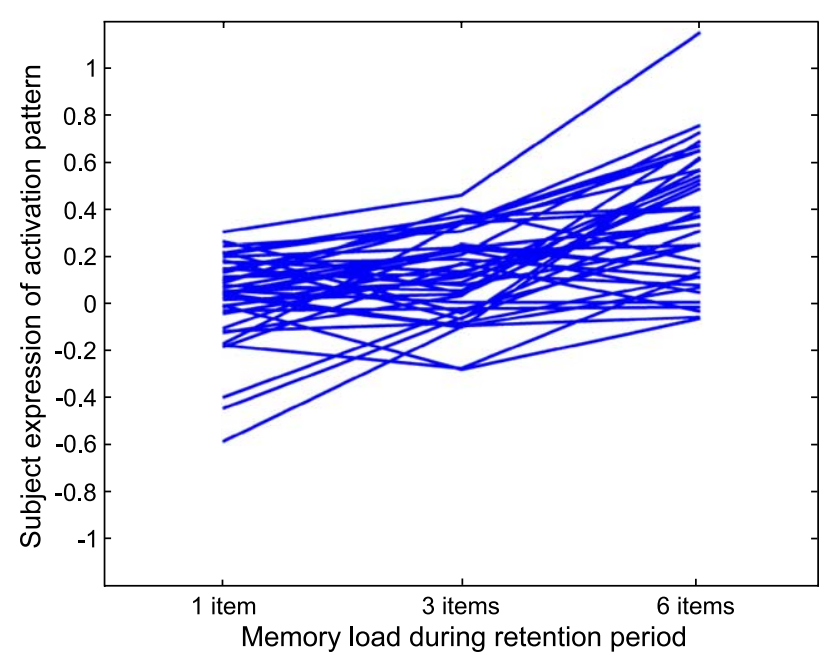

Fig. 2. Subject expression of the activation pattern resulting from the first two principal components of the Ordinal Trend Canonical Variates Analysis. Expression of the covariance pattern increased monotonically across memory load (permutation test: $\mathrm{rm}-F=38.6575, p<0.001$ ). 
cerebellum. Decreasing activation was found in the occipito-temporal lobe (BA 19,39,22), insula (BA 13) as well as the medial prefrontal cortex (BA 9,10) and limbic areas (BA 24,33; see Table 2; Figs. 3 and 4).

We then tested whether SSF correlated with individuals' behavioral performance or neuropsychological measures recorded outside the scanner. The correlation with the memory-load-related increase in reaction times was statistically significant $\left(R^{2}=0.12, p<0.05\right)$. There was also a significant negative correlation with NARTIQ $\left(R^{2}=0.15\right.$, $p<0.05)$ but no correlation with load-related increases in reaction time variability (Fig. 5).

The higher the SSF of the load-related network, the lower subjects' NARTIQ and the slower they responded to the probe at test. NARTIQ and the load-related reaction time increase themselves, although were not correlated $\left(R^{2}=0.02\right.$, $p=0.3816$ ).

The correlation of the activation pattern's SSF with behavior and neuropsychological variables hints at the greater efficiency of subjects with higher NARTIQ compared with subjects of lower NARTIQ: the latter had to employ the load-related network to a greater degree during retention with a subsequent slowing of their responses to the probe item.

We also obtained an activation pattern as a linear combination of the first eight principal components of the probe phase data that showed a monotonic increase in expression across set size (permutation test: $\mathrm{rm}-F=23.1348$, $p<0.001)$. This pattern accounted for $5 \%$ of the variance in the data. Areas of increasing activation across set size were found in the bilateral cerebellum, left lateral PFC (BA

Table 2

Talairach locations of brain regions with significant activation or deactivation across load levels during the retention phase, as ascertained by a bootstrapresampling test $(|z|>2.33)$

\begin{tabular}{|c|c|c|c|c|c|}
\hline$X$ & Y & $Z$ & Lobe & Structure & Brodmann label \\
\hline \multicolumn{6}{|c|}{ Positive weights } \\
\hline 14 & -68 & -10 & Cerebellum & Culmen & * \\
\hline 26 & -63 & -15 & Cerebellum & Declive & * \\
\hline 34 & -53 & -19 & Cerebellum & Culmen & $*$ \\
\hline-53 & 10 & 7 & Frontal lobe & Precentral gyrus & Brodmann area 44 \\
\hline-6 & 1 & 63 & Frontal lobe & Superior frontal gyrus & Brodmann area 6 \\
\hline 6 & 3 & 59 & Frontal lobe & Medial frontal gyrus & Brodmann area 6 \\
\hline-51 & -31 & 44 & Parietal lobe & Inferior parietal Lobule & Brodmann area 40 \\
\hline-36 & -50 & 45 & Parietal lobe & Inferior parietal Lobule & Brodmann area 40 \\
\hline 38 & 31 & 32 & Frontal lobe & Middle frontal gyrus & Brodmann area 9 \\
\hline 38 & 44 & 24 & Frontal lobe & Middle frontal gyrus & Brodmann area 10 \\
\hline 24 & 5 & 57 & Frontal lobe & Sub-Gyral & Brodmann area 6 \\
\hline 38 & -43 & 43 & Parietal lobe & Inferior parietal lobule & Brodmann area 40 \\
\hline 51 & -37 & 44 & Parietal lobe & Inferior parietal lobule & Brodmann area 40 \\
\hline 42 & -37 & 46 & Parietal lobe & Inferior parietal lobule & Brodmann area 40 \\
\hline 44 & -8 & -10 & Temporal lobe & Sub-Gyral & Brodmann area 21 \\
\hline-24 & 3 & 55 & Frontal lobe & Sub-Gyral & Brodmann area 6 \\
\hline-32 & -63 & -17 & Cerebellum & Declive & * \\
\hline 44 & 2 & 50 & Frontal lobe & Middle frontal gyrus & Brodmann area 6 \\
\hline 0 & 20 & 49 & Frontal lobe & Superior frontal gyrus & $*$ \\
\hline-18 & 13 & -11 & Frontal lobe & Subcallosal gyrus & Brodmann area 13 \\
\hline 8 & 19 & 38 & Limbic lobe & Cingulate gyrus & Brodmann area 32 \\
\hline-59 & -31 & 3 & Temporal lobe & Middle temporal gyrus & Brodmann area 22 \\
\hline \multicolumn{6}{|c|}{ Negative weights } \\
\hline 4 & 58 & -1 & Frontal lobe & Superior frontal gyrus & * \\
\hline 2 & 49 & 18 & Frontal lobe & Medial frontal gyrus & * \\
\hline 10 & -63 & 31 & Parietal lobe & Precuneus & $*$ \\
\hline-8 & -59 & 29 & Occipital lobe & Precuneus & Brodmann area 31 \\
\hline-51 & -57 & 23 & Temporal lobe & Superior temporal gyrus & Brodmann area 39 \\
\hline-42 & -66 & 40 & Parietal lobe & Inferior parietal lobule & Brodmann area 39 \\
\hline-46 & -12 & 1 & Temporal lobe & Superior temporal gyrus & $*$ \\
\hline 63 & -50 & 15 & Temporal lobe & Superior temporal gyrus & Brodmann area 22 \\
\hline-4 & -23 & 38 & Limbic lobe & Cingulate gyrus & $*$ \\
\hline-22 & 35 & 42 & Frontal lobe & Middle frontal gyrus & Brodmann area 8 \\
\hline-2 & 44 & 33 & Frontal lobe & Medial frontal gyrus & Brodmann area 9 \\
\hline 40 & -9 & 15 & Sublobar & Insula & Brodmann area 13 \\
\hline-2 & 20 & 21 & Limbic lobe & Anterior cingulate & Brodmann area 33 \\
\hline 4 & 19 & 25 & Limbic lobe & Anterior cingulate & Brodmann area 24 \\
\hline
\end{tabular}

(Talairach Daemon Client 1.1, Research Imaging Center, University of Texas Health Science Center at San Antonio). White matter locations have been removed. Top: positive weights, indicating activation across load levels for the majority of subjects; bottom: negative weights, indicating deactivation. 

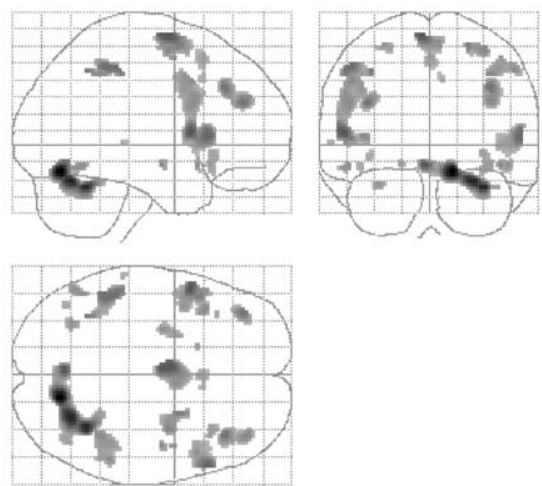

SPMresults:

Height threshold $\mathrm{T}=2.33$

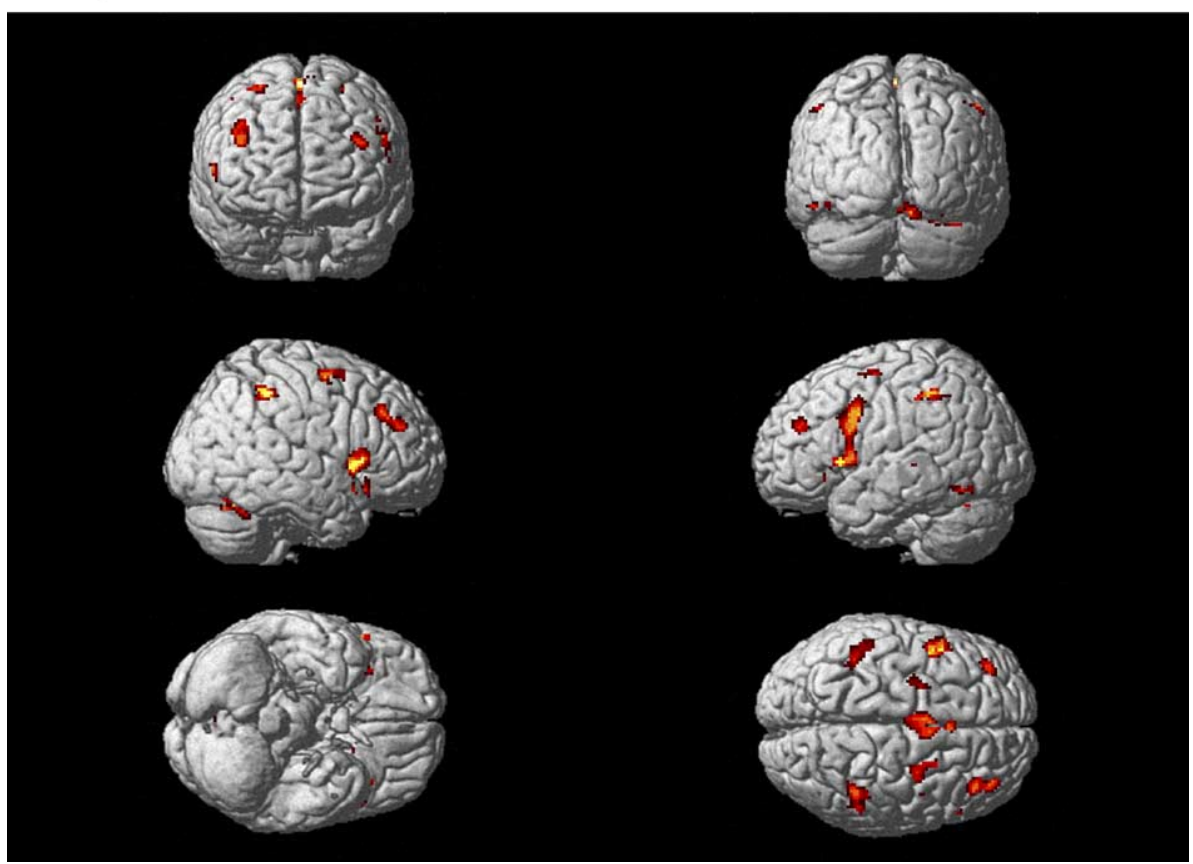

Fig. 3. Upper half: axial, coronal, and sagittal glass-brain projections of brain regions that have positive weights in the activation pattern, i.e., whose associated activation increases for most people across memory load. The threshold of the $z$-statistic was $z>2.33$. Lower half: surface-rendered projections of the regions displayed in the glass-brain projections.

9,46,47), and thalamus. Areas decreasing in activation were more widely distributed and located in parietal, frontal, temporal, and limbic lobes as well as basal ganglia (see detailed listing in Table 3). The load-related increase in subject expression of this activation pattern could account for the increase in reaction time variability: the more subject increased their expression from 1 to 6 , the more variable they became in their reaction time as well $\left(R^{2}=0.16, p<0.01\right.$; graph not shown; the other two load-related SSF increases, 6-3 and 3-1, did not yield a correlation between pattern expression and reaction time variability). The load-related increase correlated negatively with NARTIQ $\left(R^{2}=0.14\right.$, $p<0.05$ ), although this correlation is brought about by one overly influential subject outlier and therefore only affords diminished confidence in the robustness of the relationship. No significant correlation with the reaction time increase from 1 to 6 was found in this pattern's SSF.
We also investigated whether the load-related increases in subject expression of the three activation patterns were correlated, but there was no significant correlation between any of the three task phases. This means, for instance, that a subject's increase in the expression of the pattern discerned from the data of the retention phase does not give any indication of the magnitude of the change in expression of the pattern obtained from the probe period. This confirmssimilar to the processing logic in the original Sternberg task [31,32] - that the load-related neural activation patterns are operating independently from each other in different epochs of our Sternberg-task version. Also, trying to account for the variance in the three outcome measures, (1) mean reaction time, (2) variability in reaction time, and (3) NARTIQ, with the combined load-related expression differences of all three activation patterns in a linear regression were no more successful than predicting for each of the dependent 

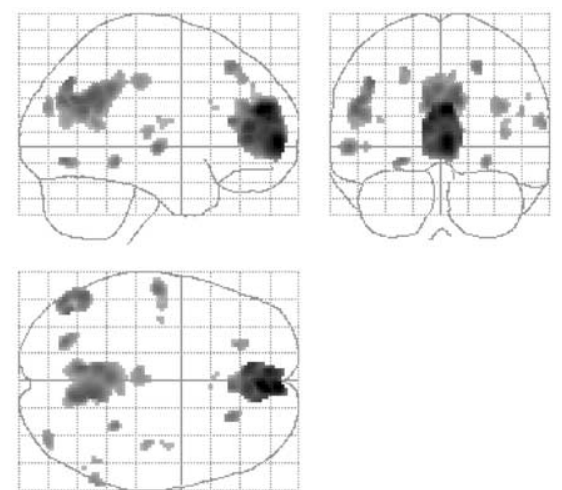

SPMresults:

Height threshold $\mathrm{T}=2.33$

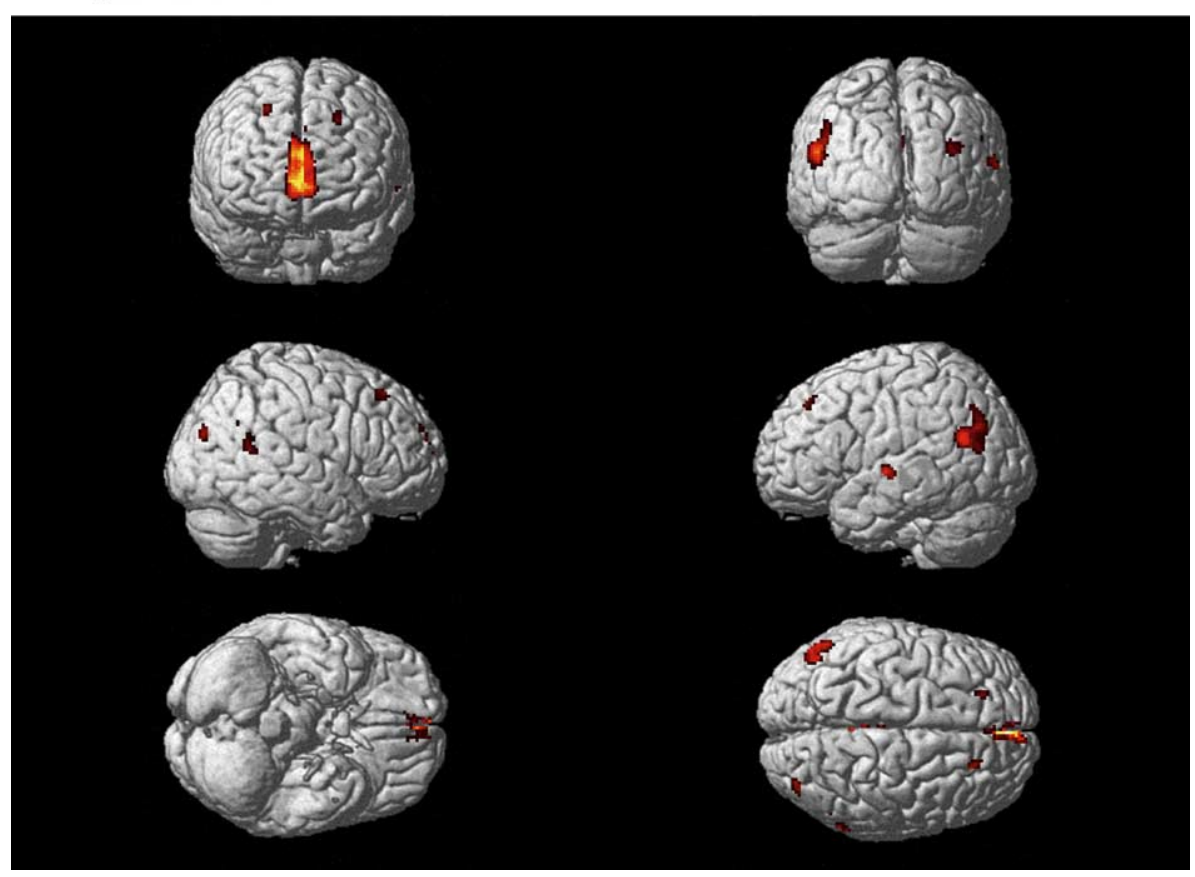

Fig. 4. Upper half: axial, coronal, and sagittal glass-brain projections of brain regions that have negative weights in the activation pattern, i.e., whose associated activation decreases for most subjects across memory load. The threshold the $z$-statistic was $z<-2.33$. Lower half: surface-rendered projections of the regions displayed in the glass-brain projections.
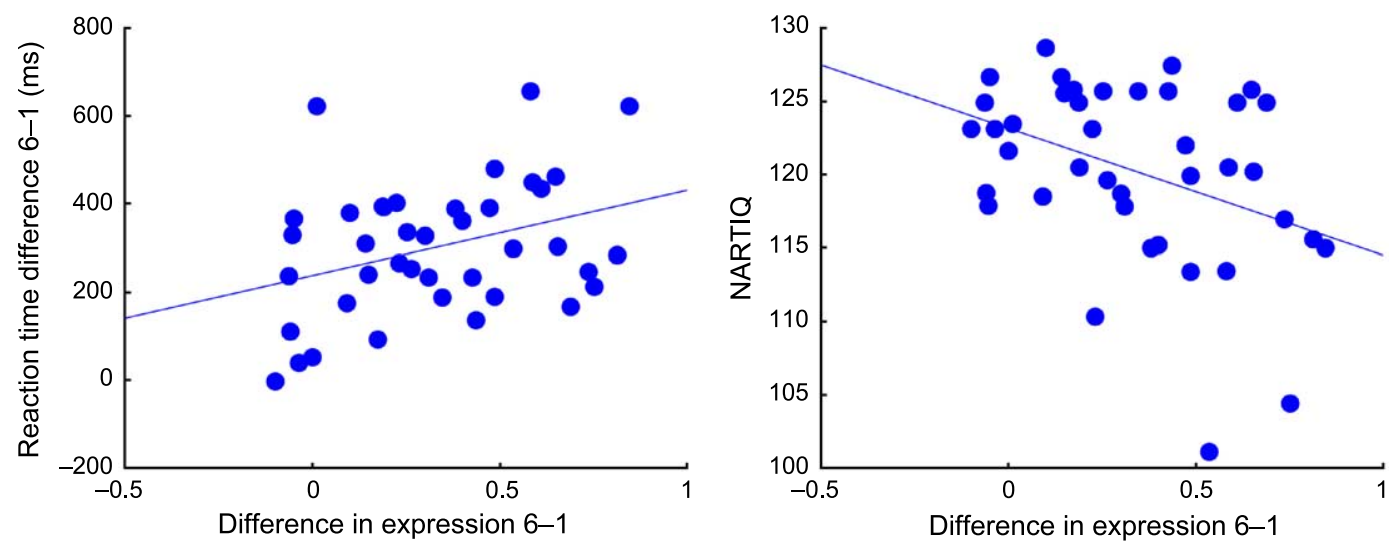

Fig. 5. Left: positive correlation $\left(R^{2}=0.12, p<0.05\right)$ between the increase in the load-related network's expression and the reaction time increase from the rehearsal of 1 to 6 items. Right: negative correlation $\left(R^{2}=0.15, p<0.05\right)$ between the increase in expression and NARTIQ. The more the subject increased their expression of the load-related network from the rehearsal of 1 to 6 items, the slower they became when responding to the probe at test and the lower their NARTIQ. NARTIQ and the reaction time increase themselves were not correlated $\left(R^{2}=0.02, p=0.3816\right)$. 
Table 3

Talairach locations of brain regions with significant activation or deactivation across load levels during the probe phase, as ascertained by a bootstrapresampling test $(|z|>2.33)$

\begin{tabular}{|c|c|c|c|c|c|}
\hline$X$ & Y & $z$ & Lobe & Structure & Brodmann label \\
\hline \multicolumn{6}{|c|}{ Positive weights } \\
\hline 18 & -67 & -15 & Cerebellum & Declive & * \\
\hline-12 & -49 & -16 & Cerebellum & Culmen & * \\
\hline-4 & -73 & -13 & Cerebellum & Declive & $*$ \\
\hline-51 & 7 & 31 & Frontal lobe & Inferior frontal gyrus & Brodmann area 9 \\
\hline 38 & 16 & -1 & Sublobar & Insula & Brodmann area 13 \\
\hline-34 & -63 & -20 & Cerebellum & Declive & $*$ \\
\hline-38 & -54 & -29 & Cerebellum & Culmen & $*$ \\
\hline-6 & -19 & 12 & Sublobar & Thalamus & Medial Dorsal Nucleus \\
\hline-46 & 39 & 13 & Frontal lobe & Inferior frontal gyrus & Brodmann area 46 \\
\hline-44 & 13 & -4 & Frontal lobe & Inferior frontal gyrus & Brodmann area 47 \\
\hline \multicolumn{6}{|c|}{ Negative weights } \\
\hline-6 & -52 & 45 & Parietal lobe & Precuneus & * \\
\hline-2 & -59 & 29 & Limbic lobe & Cingulate gyrus & Brodmann area 31 \\
\hline-20 & 39 & 39 & Frontal lobe & Superior frontal gyrus & Brodmann area 8 \\
\hline 8 & 41 & 2 & Limbic lobe & Anterior cingulate & Brodmann area 32 \\
\hline-46 & -59 & 23 & Temporal lobe & Middle temporal gyrus & Brodmann area 39 \\
\hline-6 & 37 & -2 & Limbic lobe & Anterior cingulate & Brodmann area 24 \\
\hline-12 & 19 & -1 & Sublobar & Caudate & Caudate head \\
\hline 22 & 38 & -17 & Frontal lobe & Middle frontal gyrus & Brodmann area 11 \\
\hline 26 & 20 & 43 & Frontal lobe & Middle frontal gyrus & Brodmann area 8 \\
\hline-10 & 16 & 7 & Sublobar & Caudate & Caudate body \\
\hline-28 & -43 & -5 & Limbic lobe & Parahippocampal gyrus & Brodmann area 19 \\
\hline 26 & 33 & 44 & Frontal Lobe & Superior Frontal gyrus & $*$ \\
\hline
\end{tabular}

White matter locations have been removed. Top: positive weights, indicating activation across load levels for the majority of subjects; bottom: negative weights, indicating deactivation.

variables with a single activation pattern as mentioned before: NARTIQ and mean $6-1$ reaction time difference are best explained by the 6-1 SSF difference of the load-related pattern in the retention period, whereas the 6-1 increase in reaction time variability is best explained by the load-related pattern in the probe period.

\section{Discussion}

We successfully identified load-related activation patterns in the data from all task phases (stimulus, retention, probe) of a delayed-match-to-sample task. The majority of subjects in our sample $(>20)$ displayed increasing SSFs of these activation patterns with increasing numbers of items that had to be retained. That single covariance patterns capture the neural response across several conditions implies that the correlative relationships between all brain regions' activation behaviors are fixed. Some brain regions decrease their activity, while others increase their activity in response to increasing memory load. The relationships between these increases and decreases are given by the loadings in the covariance patterns and do not change in response to increasing memory load themselves. Fixed interregional relationships indicate sustained functional connectivity; the inferential framework in our analysis method ensures that such sustained functional connectivity is identified with high specificity [14]. However, interre- gional correlations were not measured explicitly for each memory-load separately, and subsequently no direct comparison of these correlations was performed. It is conceivable that the raw data, i.e., the superposition of the loadrelated covariance patterns and additional noise-sources, give rise to interregional correlations that might change with memory load. We did not pursue this possibility in this report.

While the activation pattern found in the encoding phase could not account for any behavioral performance or neuropsychological variables, the other two activation patterns could. The increase in the activation pattern's SSF from the 1-item to the 6-item retention phase correlated negatively with NARTIQ and positively with the corresponding increase in the (within-subject mean) reaction times. Increase in expression for the probe phase pattern predicted the increase in (within-subject) reaction time variability from the 1 -item to the 6 -item condition. These correlations with behavioral and neuropsychological variables were obtained with our multivariate analysis technique without having incorporated any information or hypotheses about brain-behavior relationships. Only information about the experimental design, i.e., parametric changes in memory load (number of items to be studied, remembered, and retrieved), was included in the functional imaging analysis.

The areas exhibiting activation and deactivation across memory load in the stimulus phase are plausible in terms of visual encoding processes and include occipital, parietal, 
temporal, and frontal sites. Increased visual spatial-attentional processing demands $[4,16,29]$ would be consistent with some of the occipito-fronto-parietal areas shown to activate across item numbers like precuneus (BA 7), preSMA (BA 6), lingual gyrus, and thalamus. We found both parahippocampal activation as well as deactivation which partly parallels the activation observed during the encoding phase of previous episodic and working memory studies $[15,24,25]$. The larger set of areas that deactivate across the number of study items contains some of the same structures just mentioned and is more difficult to relate to other studies, since deactivation have been reported less often than activations. Because the activation pattern obtained during the stimulus phase did not correlate with any aspect of behavior or NARTIQ, it is difficult to separate the part of its neuroanatomic make-up that is genuinely related to the encoding of the study items from other parts, which might not be important for the performance of the task, but nevertheless display a monotonic relationship to the number of items on display. Short of carrying out a redesigned experiment, it may be possible to model the different latent processing components and their influence on specific aspects of performance as a means to differentiate the neural correlates of these individual processes. Although canonical variate analysis, and ordinal trend analysis in particular, can be used to an advantage in this aspect of network modeling, we have not pursued these extensions of ordinal trend analysis here.

The load-related pattern in the retention phase afforded a significant correlation with the subjects' reaction times during the probe phase as well as NARTIQ. We find this link between neural activation and subsequent behavior as well as innate ability our strongest result of the report: the more subjects increased their pattern expression from the 1item to the 6-item conditions during retention, the more they slowed in their responses at probe and the lower their NARTIQ. This finding is consistent with a previous report [28] that conducted a thorough analysis of task- and subjectfactors in a working memory paradigm similar to ours, with particular emphasis on the lateral PFC. Also similar to the cited report [28], we found load-related activation of lateral PFC (BA 9,44), reproducing the effect of task demand, while subjects with faster responses (and in our case: higher NARTIQ) activate these areas less. ${ }^{1}$ Apparently, the covariance pattern obtained during retention captures a necessary aspect of neural processing that, while displaying a relationship with behavioral outcomes, is not advantageous for task performance. Greater utilization of the covariance pattern did not result in better, but worse,

\footnotetext{
${ }^{1}$ In the same vein, a similar statement can be made about the activation of lateral PFC during the probe phase: similarly to Ref. [28], we also found lateral PFC activity that increased in response to increasing memory demand with the slight difference that now, people became less variable in their reaction time (as opposed to slower) the less they activated lateral PFC.
}

performance. Also, subjects who employed the covariance pattern more had lower NARTIQ. It may be that that betterperforming subjects change their strategies (hence, changing the constellation of activated regions) with increasing memory loads. Similar to well-established findings with older adults in verbal learning [6], subjects who do not perform so well in this task may persist in performing the task, using the same strategy. A further hint that there are substantial individual differences in rehearsal when compared to the encoding phase is the reduced amount of variance the rehearsal pattern accounts for in comparison to the encoding pattern, i.e., $9 \%$ vs. $20 \%$, respectively.

Areas that correlate positively with the memory-load related increase in activation were the cerebellum, the inferior parietal lobe (BA 40), and the left middle temporal gyrus (BA 22); the latter region merits speculation that auditory rehearsal is taking place during the retention period [1]. The areas that deactivate with increasing memory demand during the retention period have weights that are as big or bigger than the areas of activation. Deactivations with a task parameter have received more attention in recent years and offer some points of contact with our results. Our results showed a robust deactivation of the anterior cingulate gyrus and the medial prefrontal cortex. The specific neuroanatomy of connections between medial and lateral prefrontal cortices as well as other cortical areas is an area of ongoing research [2,3] that posits that the connectivity among these particular regions of $\mathrm{PFC}$ and posterior regions (involved in oculomotor guidance and spatial attention) contributes to the synthesis of memory, cognition, and emotion in general. A recent study using working-memory with an $N$-back design [22] also found anterior medial prefrontal deactivating with increasing memory load. The authors of this study offer a rationale for the deactivation of the medial prefrontal cortex that is consistent with the general resource account framework [9] mentioned in the Introduction. Because of the role of prefrontal limbic cortices (i.e., orbitofrontal and medial prefrontal cortices) in emotional processing [2], the results suggest a shifting balance during higher cognitive processing which manifests itself in increasing activity in cortical cognitive areas and decreasing activity in the limbic and paralimbic structures. Such reciprocal changes in brain activation associated with emotional and cognitive processing are also found in mood disorders such as depression [17], although with a different relative sign to our findings. In depressed patients, hyperactivity in limbic and paralimbic areas are accompanied by decreased activity in cortical areas, resulting in worse cognitive performance. Negative mood and high memory demand can thus be interpreted as opposite ends of a common continuum.

The probe phase produced a load-related activation pattern that predicted the increase in reaction time variability in subjects' responses. In contrast to the stimulus and retention phases, which revealed load-related behavior 
within the first two principal components, this pattern required a linear combination of the first eight principal components and only accounted for $5 \%$ of the variance, signaling the greatest intersubject variability of all task phases. This variability might be attributable to the loadindependent cognitive processes that are present during the probe period in addition to memory scanning and might introduce substantial intersubject variability: subjects have to visually process the probe letter and make a decision about the match, select a response, and press a button. A temporal separation of these processes similar to the experimental design in Ref. [26] might allow a better disentanglement of load-related and unrelated component processes. For completeness, we note that, in the comprehensive report [28] of the behavior of DLPFC in a memory task similar to ours, DLPFC was found to increase with memory load during retrieval, albeit less for more accurate and fast responders.

We also mention that our experimental design already contained one factor that was collapsed over in our analysis but theoretically allows a deeper analysis of the binary decision process: the type of probe the subject has to respond to, i.e., true positive or negative. It is conceivable that there are differences between the memory scanning and decision processes for these different probe types, which will also be reflected by two distinct probetype specific neural activation patterns. Modeling these probe-type specific activation patterns separately might show topographic differences and yield better brainbehavior correlations of these patterns with their respective probe-specific reaction times. However, because only half the number of epochs would be available for modeling each probe type, the neural responses would also be more variable. For the current report, the goal was to find neural activation patterns and brain-behavior correlation regardless of probe type - this factor was therefore collapsed over in our analysis.

The results from retention and probe phase indicate that the speed of subjects' responses is less related to their neural activation pattern at the time of the response (nor at the time of encoding) than to their neural activation pattern during the preceding retention period. The question therefore arises as to how the MR signal related to cognitive processing prior to the onset of the probe can correlate with the reaction time, which is measured subsequently to the probe onset. McClelland's cascade model of cognitive processing [18] provides one possible mechanism. In this case, a cognitive process can affect subsequent processing in two ways, i.e., the speed in which it reaches completion and the integrity of the information passed on to the next stage. We propose that the rehearsal mechanism varies in integrity between individuals, yielding a stimulus set available for comparison to the probe that varies in quality. This has two consequences. First, rehearsal with poorer integrity leads to a slower comparison between probe and each stimulus item. This in turn relates maintenance activity to stimulus set-size and memory scan speed. Second, poorer maintenance integrity leads to more effort on the part of subjects. This effort is reflected in a "ramping up" of the brain network supporting maintenance and a larger MR signal associated with that network.

Interestingly, a greater need for rehearsal during the retention period signals lower intelligence, which is consistent with other recent reports [13,30] where subjects' innate ability or "cognitive reserve" can account for observed neural activation patterns. During the probe phase on the other hand, subject's brain activation during button press was only predictive of reaction time variability, not reaction time. This dissociation between the different behavioral correlates of load-related neural processing in retention and probe is also reflected in the lack of a significant correlation between all task phases' respective load-related patterns' increases in expression from 1 to 6 . Such changes in expression of the loadrelated pattern of one task phase therefore give no indication of the change in expression of the load-related pattern in a different task phase, upstream or downstream alike.

We conclude this report with the observation that employment of our load-related activation patterns, when predictive of behavioral outcome measures or NARTIQ, was deleterious for task performance. Subjects who were less efficient and manifested a larger increase in their expression of the activation patterns during retention and probe showed diminished behavioral performance and lower NARTIQ. This is similar to well-established findings in the aging literature [6], where older individuals were found to persist in the usage of ineffective strategies for verbal working memory, while better-performing subjects might change theirs. In our task, for good performers, this might hint at different covariance patterns becoming operational for increasing memory loads.

These additional latent activation patterns, whose increasing expression is beneficial for task performance, might be present in the data but might not account for enough variance to enable detection in the multivariate analysis. This possibility calls for more sophisticated design matrices that utilize behavioral outcome measures and experimental design information simultaneously, which will be the topic of future reports. In this report, we made the first step and isolated the necessary but not sufficient neural network correlates of memory load in a working memory task, some of which could also account for behavioral outcome variables as well as NARTIQ.

\section{Acknowledgments}

This work was supported by the Defense Advanced Research Projects Agency (DARPA) grant DAAD 19-0201-01147. We thank two anonymous reviewers for helpful suggestions that improved the manuscript. 


\section{References}

[1] A. Baddeley, Working memory: looking back and looking forward, Nat. Rev., Neurosci. 4 (2003) 829-839.

[2] H. Barbas, Connections underlying the synthesis of cognition, memory, and emotion in primate prefrontal cortices, Brain Res. Bull. 52 (2000) 319-330.

[3] H. Barbas, H. Ghashghaei, S.M. Dombrowski, N.L. Rempel-Clower, Medial prefrontal cortices are unified by common connections with superior temporal cortices and distinguished by input from memoryrelated areas in the rhesus monkey, J. Comp. Neurol. 410 (1999) $343-367$.

[4] M.S. Beauchamp, L. Petit, T.M. Ellmore, J. Ingeholm, J.V. Haxby, A parametric fMRI study of overt and covert shifts of visuospatial attention, Neuroimage 14 (2001) 310-321.

[5] N. Cowan, The magical number 4 in short-term memory: a reconsideration of mental storage capacity, Behav. Brain Sci. 24 (2001) 87-114 (discussion 114-85).

[6] F.I.M. Craik, J.C. Rabinowitz, Age differences in the acquisition and use of verbal information: a tutorial review, in: H. Bouma, D.G. Bouwhuis (Eds.), Attention and Performance, X, Erlbaum, Hillsdale, NJ, 1984.

[7] M. D’Esposito, B.R. Postle, B. Rypma, Prefrontal cortical contributions to working memory: evidence from event-related fMRI studies, Exp. Brain Res. 133 (2000) 3-11.

[8] B. Efron, R.J. Tibshirani, An Introduction to the Bootstrap, CRC Press, LLC, New York, 1994, 436 pp.

[9] R.W. Engle, A.R.A. Conway, S.W. Tuholsky, R.J. Shisler, A resource account of inhibition, Psychol. Sci. 6 (1995) 122-125.

[10] K.J. Friston, P. Fletcher, O. Josephs, A. Holmes, M.D. Rugg, R. Turner, Event-related fMRI: characterizing differential responses, Neuroimage 7 (1998) 30-40.

[11] K.J. Friston, O. Josephs, E. Zarahn, A.P. Holmes, S. Rouquette, J. Poline, To smooth or not to smooth? Bias and efficiency in fMRI timeseries analysis, Neuroimage 12 (2000) 196-208.

[12] C.G. Habeck, Y. Stern, H.B. Posner, J.R. Moeller, Regional covariance analysis of event-related fMRI, SFN Abstracts, (2002).

[13] C. Habeck, H.J. Hilton, E. Zarahn, J. Flynn, J. Moeller, Y. Stern, Relation of cognitive reserve and task performance to expression of regional covariance networks in an event-related fMRI study of nonverbal memory, Neuroimage 20 (2003) 1723-1733.

[14] C.G. Habeck, J.W. Krakauer, C. Ghez, Y. Stern, H.A. Sackeim, D. Eidelberg, J.R. Moeller, A new approach to spatial covariance modeling of functional brain imaging data: ordinal trend analysis, Neural Computation (under review).

[15] S. Kohler, M. Moscovitch, G. Winocur, A.R. McIntosh, Episodic encoding and recognition of pictures and words: role of the human medial temporal lobes, Acta Psychol. (Amst.) 105 (2000) 159-179.

[16] K.S. LaBar, D.R. Gitelman, T.B. Parrish, M. Mesulam, Neuroanatomic overlap of working memory and spatial attention networks: a functional MRI comparison within subjects, Neuroimage 10 (1999) 695-704.

[17] H.S. Mayberg, M. Liotti, S.K. Brannan, S. McGinnis, R.K. Mahurin, P.A. Jerabek, J.A. Silva, J.L. Tekell, C.C. Martin, J.L. Lancaster, P.T.
Fox, Reciprocal limbic-cortical function and negative mood: converging PET findings in depression and normal sadness, Am. J. Psychiatry 156 (1999) 675-682.

[18] J.L. McClelland, On the time relations of mental processes: an examination of systems of processes in cascade, Psychol. Rev. 86 (1979) 287-324.

[19] A.R. McIntosh, Mapping cognition to the brain through neural interactions, Memory 7 (1999) 523-548.

[20] A.R. McIntosh, F.L. Bookstein, J.V. Haxby, C.L. Grady, Spatial pattern analysis of functional brain images using partial least squares, Neuroimage 3 (1996) 143-157.

[21] A.R. McIntosh, N.J. Lobaugh, R. Cabeza, F.L. Bookstein, S. Houle, Convergence of neural systems processing stimulus associations and coordinating motor responses, Cereb. Cortex 8 (1998) 648-659.

[22] J.B. Pochon, R. Levy, P. Fossati, S. Lehericy, J.B. Poline, B. Pillon, D. Le Bihan, B. Dubois, The neural system that bridges reward and cognition in humans: an fMRI study, Proc. Natl. Acad. Sci. U. S. A. 99 (2002) 5669-5674.

[23] B.R. Postle, E. Zarahn, M. D’Esposito, Using event-related fMRI to assess delay-period activity during performance of spatial and nonspatial working memory tasks, Brain Res. Brain Res. Protoc. 5 (2000) $57-66$.

[24] C. Ranganath, M. D’Esposito, Medial temporal lobe activity associated with active maintenance of novel information, Neuron 31 (2001) $865-873$.

[25] S.A. Rombouts, F. Barkhof, M.P. Witter, W.C. Machielsen, P. Scheltens, Anterior medial temporal lobe activation during attempted retrieval of encoded visuospatial scenes: an event-related fMRI study, Neuroimage 14 (2001) 67-76.

[26] J.B. Rowe, I. Toni, O. Josephs, R.S. Frackowiak, R.E. Passingham, The prefrontal cortex: response selection or maintenance within working memory? Science 288 (2000) 1656-1660.

[27] B. Rypma, V. Prabhakaran, J.E. Desmond, G.H. Glover, J.D. Gabrieli, Load-dependent roles of frontal brain regions in the maintenance of working memory, Neuroimage 9 (1999) 216-226.

[28] B. Rypma, J.S. Berger, M. D'Esposito, The influence of workingmemory demand and subject performance on prefrontal cortical activity, J. Cogn. Neurosci. 14 (2002) 721-731.

[29] E.R. Simon-Thomas, K. Brodsky, C. Willing, R. Sinha, R.T. Knight, Distributed neural activity during object, spatial and integrated processing in humans, Brain Res. Cogn. Brain Res. 16 (2003) 457-467.

[30] Y. Stern, E. Zarahn, H.J. Hilton, J. Flynn, R. DeLaPaz, B. Rakitin, Exploring the neural basis of cognitive reserve, J. Clin. Exp. Neuropsychol. 25 (2003) 691-701.

[31] S. Sternberg, High-speed scanning in human memory, Science 153 (1966) 652-654.

[32] S. Sternberg, Memory-scanning: mental processes revealed by reaction-time experiments, Am. Sci. 57 (1969) 421-457.

[33] K.J. Worsley, J.B. Poline, K.J. Friston, A.C. Evans, Characterizing the response of PET and fMRI data using multivariate linear models, Neuroimage 6 (1997) 305-319.

[34] E. Zarahn, Testing for neural responses during temporal components of trials with BOLD fMRI, Neuroimage 11 (2000) 783-796. 